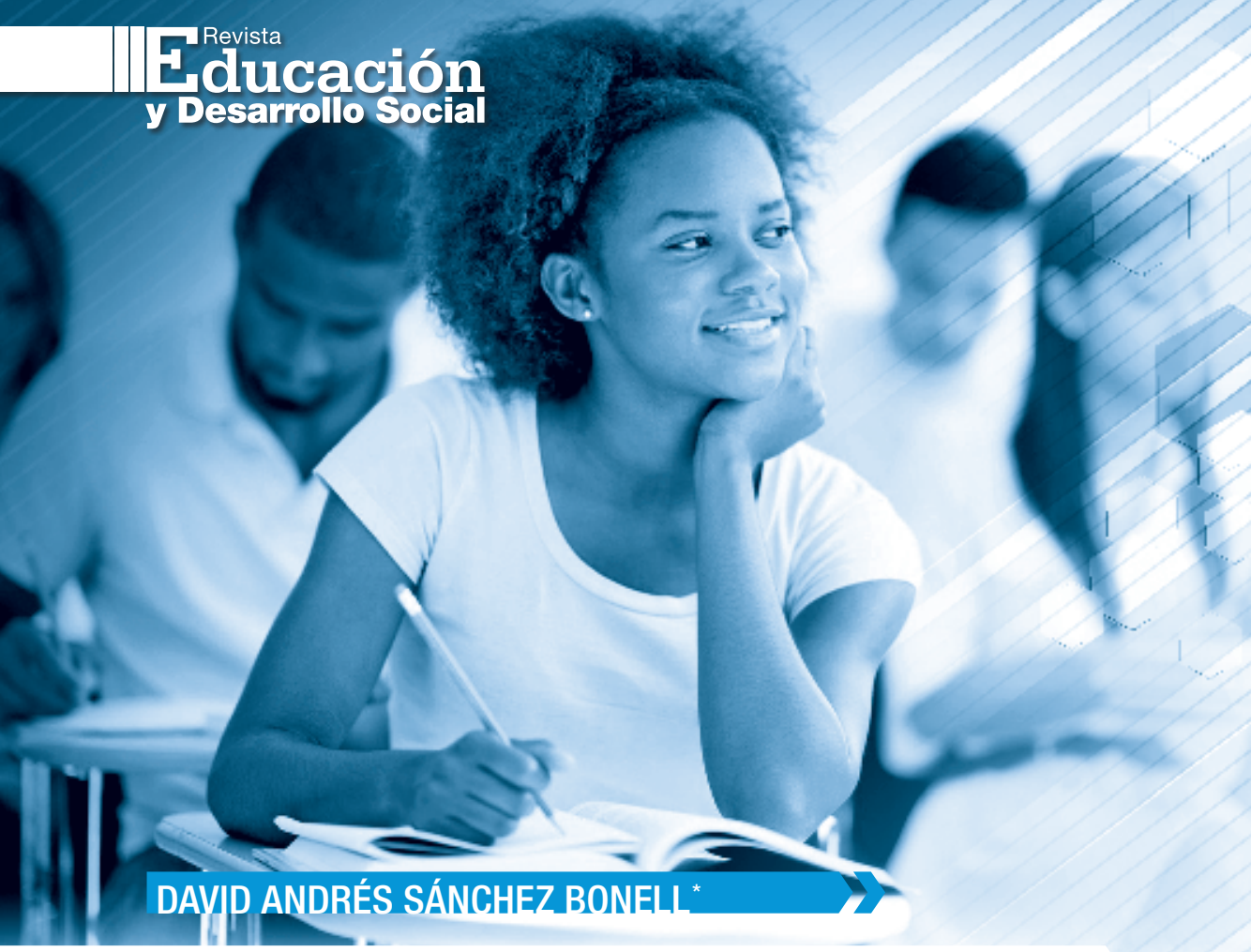

FECHA DE RECEPCIÓN: 21 DE MARZO DE 2014

FECHA DE EVALUACIÓN: 14 DE MAYO DE 2014

\title{
ATENDER LA \\ INEQUIDAD EDUCATIVA: \\ ELEMENTO FUNDAMENTAL \\ PARA INCIDIR EN LA \\ MOVILIDAD SOCIAL
}

Addressing inequality in education: a key element to influence social mobility

Atender a desigualdade educativa; elemento fundamental para influenciar na mobilidade social

\footnotetext{
Docente - investigador. Magister en Ciencias U Nacional. Doctorando en educación Universidad Pedagógica Nacional. docente Instituto Alberto Merani, Universidad del Rosario y Universidad Militar Nueva Granada.
} 


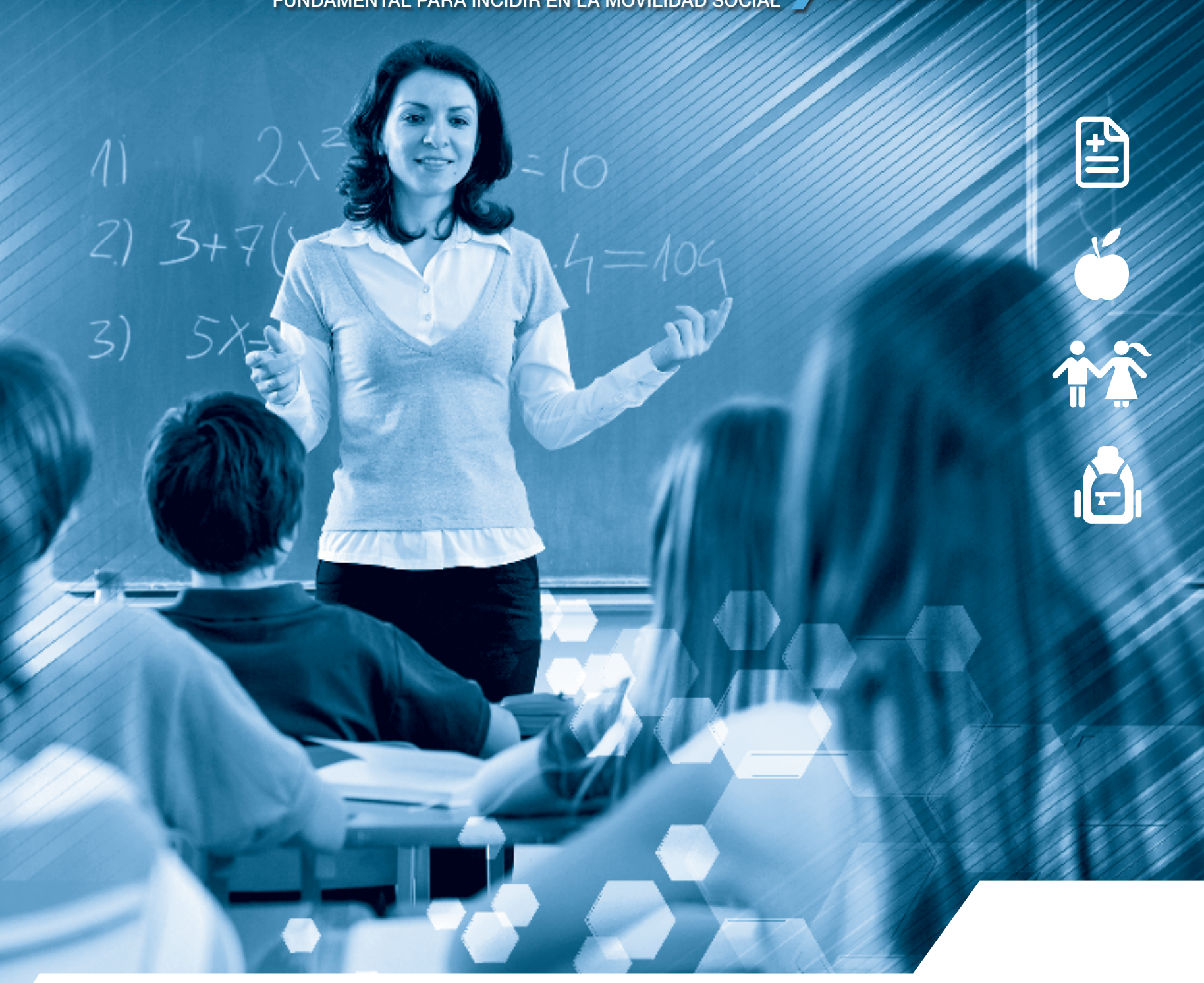

Asegurar que todos los estudiantes aprendan a niveles de excelencia requiere ajustar la enseñanza y las ayudas pedagógicas a las necesidades y características de cada uno. La educación inclusiva y la atención a la diversidad demandan una mayor competencia profesional de los docentes, un trabajo colaborativo entre éstos, las familias y los alumnos, y proyectos educativos más amplios y flexibles que favorezcan la participación y aprendizaje de todos. Exige también el desarrollo de un currículum pertinente y equilibrado en cuanto al tipo de aprendizajes que promueve, una variedad de actividades y situaciones de aprendizaje, un amplio repertorio de estrategias de enseñanza y un clima escolar en el que se acoja y valore a todos por igual, brindando más apoyo a quién más lo necesite. En definitiva, el desafío de la inclusión es avanzar hacia una educación para todos, con todos y para cada uno. (Blanco, 2088) 


\section{RESUMMN}

Colombia representa un caso suigeneris en América latina en relación con la movilidad social y la movilidad educativa, mientras en el resto de países esta depende del "estatus" socioeducativo y sociocultural de las familias, en el país además, depende del "estatus socio económico de la población en términos de cantidad, calidad y distribución de ingresos. Lo paradójico es que en Colombia la economía no solo es fuerte con respecto al resto de los países sino que viene creciendo de manera importante, esto supondría una alta movilidad social y posiblemente una alta movilidad educativa, ¿Por qué entonces es tan baja la movilidad social y educativa en el país? ¿Cuáles son las políticas acciones y directrices que deben ser tenidas en cuenta para atender esta problemática?, ¿Dónde radica la inequidad del sistema educativo?, existen brechas en cada uno de los niveles del sistema educativo, son marcadas las diferencias de calidad acceso, cobertura y permanencia en diferentes sectores de sistema educativo y aun son marcadas las diferencias en la formación por géneros así como los resultados obtenidos en competencias básicas.

Palabras claves: Inequidad, movilidad social, movilidad educativa, acceso, cobertura, equidad calidad, obtención de logro, política de equidad.

\section{ABSTRAGT}

Colombia is a case sui generis in Latin America, as for social and educational mobility. While in other countries that depends on the social, educational and cultural status of the families, in
Colombia it also depends on the social and economical status of population in terms of quantity, quality and distribution of income. The paradox is that in Colombian economy is not only strong compared to the rest of the countries but it has had an important growth which would mean great social mobility and, possibly, great education mobility. Then ¿Why is there such a low social and education mobility in the country? ¿What are the policies, actions and guidelines that need to be taken into account to address these problems? ¿Where does inequality of the education system lie in? There are gaps in each of the levels of the education system, there are marked differences in quality, access, coverage and continuity in different sectors of the education system and the difference in training by gender are still marked, as well as the results obtained in basic competencies.

Keywords: Inequality, Social Mobility, Educational Mobility, Access, Coverage, Equality, Quality, Achievement, Equality Policy.

\section{RESUMMO}

A Colômbia representa um caso sui generis na América Latina em relação à mobilidade social e a mobilidade educativa, no entanto em outros países, isso depende do "status" socioeducativo e sociocultural das famílias, no país também depende do "status" socioeconômico da população em termos de quantidade, qualidade e distribuição de renda. O paradoxo é que na Colômbia a economia não sómente é forte em relação a outros países, mas está crescendo significativamente, isso implicaria uma alta mobilidade social e, possivelmente, uma elevada mobilidade educacional, 
¿Por que então é tão baixa a mobilidade social e educativa no país? ¿Quais são as ações, políticas e diretrizes que devem ser considerados para resolver este problema? ¿Onde está a desigualdade do sistema de ensino? Existem lacunas em cada um dos níveis do sistema de ensino, são acentuadas as diferenças de qualidade, acesso, cobertura e permanência nos diferentes setores do

Aceptando la invitación que nos hace el PNUD y su equipo de trabajo en Desarrollo Económico Incluyente, a propósito de discutir en el escenario educativo las relaciones que inexorablemente se establecen entre inequidad social ${ }^{1}$ e inequidad esco$\operatorname{lar}^{2}$, se precisa ahondar en los factores que inciden en la movilidad social educativa; en las posibilidades de acceso, cobertura y pertinencia educativa; en la políticas y estrategias que dentro de los sistemas escolares permitan incidir en la reducción de la inequidad escolar y posterior inequidad social.

Esta invitación se hace extensiva a la comprensión de la inequidad dentro de los sistemas escolares. La inequidad escolar, aunque está relacionada con los factores que determina la inequidad social, requiere ser analizada fundamentalmente desde una óptica pedagógica que determine los factores asociados al desarrollo de los individuos sistema de ensino e ainda são acentuadas as diferenças na formação por gênero bem como os resultados obtidos em habilidades básicas.

Palavras-chave: Desigualdade, mobilidade social, mobilidade educativa, acesso, cobertura, equidade, qualidade, obtenção de realizações, política de eqüidade.

y de los diversos grupos humanos al interior de los sistemas educativos en cualquier nivel y región. La calidad, acceso y pertinencia de la educación como "bien social", define de alguna manera la posibilidad de ingresos, cobertura en salud y movilidad social futura de individuos y poblaciones, de igual forma, el no establecer condiciones y oportunidades equitativas en los escenarios educativos implica además de de baja movilidad social, baja movilidad académica específicamente frente a los procesos de desarrollo de habilidades, aptitudes y competencias, limita la participación y construcción social que a mediano y largo plazo genera costos educativos y posteriormente sociales, entre ellos: la desesperanza y la exclusión permanente de los sistemas escolares, de producción y de reconocimiento social, incluso estando vinculados los sujetos y los grupos al sistema mismo.

1. La invitación que hace el equipo de trabajo del PNUD en la diferenciación del concepto de inequidad frente al de desigualdad, plantea no solo tener en cuenta la disparidad de oportunidades sino además la tesis muy interesante en torno a que aunque se relacionan inequidad e igualdad en ser fines deseables, la igualdad no garantiza la equidad.

2. El termino inequidad escolar, también polisémico, podríamos inicialmente asumirlo dependiendo de la participación de los individuos y grupos en los diferentes niveles del sistema escolar, la inserción en el mismo así como los mecanismos de participación, de construcción de proyecto individual - colectivo y de los resultados que obtengan los diferentes grupos sociales incluso en igualdad de oportunidades. 
Un individuo o grupo de individuos que dentro de los sistemas escolares no desarrolle sus competencias, habilidades y talentos en forma integral está condenado al fracaso escolar y social. Puede incluso estar cobijado por políticas de inclusión y permanencia dentro del sistema educativo y a su vez, estar abandonado al interior de la escuela sin la movilidad de sus estructuras metales cognitivas, valorativas y de acción como dimensiones fundamentales. La movilidad y cambio social dependen directamente de la educación y de los sistemas escolares que propendan por verdaderas transformaciones del potencial humano en el país, en la región y en cada uno de las instituciones y niveles del sistema escolar Colombiano. La escuela y los sistemas educativos aportan en la reconfiguración de los saberes generales y específicos de los sujetos, permiten la movilidad cognitiva, afectiva y de acción de los sujetos en un contexto social pero sobre todo en el contexto escolar, le permite alcanzar grados y niveles distintos de compresión, interpretación y reproducción de saberes locales y universales que le son necesarios ya no solo para desenvolverse en el mundo sino para comprenderlo y poder tomar decisiones sobre su propio destino. Mientras las políticas, los currículos, las reflexiones pedagógicas y acciones educativas de los sistemas educativos permanezcan indolentes e incluso inocentes de esta realidad se incrementara el abandono, la inequidad y la exclusión dentro del sistema mismo. Mientras no se comprendan las necesidades educativas y de desarrollo de hombres y mujeres, niños y niñas, adolescentes y poblaciones con necesidades educativas especiales de manera integral, más allá de disposiciones administrativas y económicas, se seguirá abandonado y excluyendo pedagógicamente a todos los grupos etarios, particularmente a los más vulnerables. Mientras la escuela y la educación no comprenda la necesidad de formar individuos más equilibrados en su "ser" y "hacer" seguirán disminuyendo las oportunidades a quienes no caben en modelos universales y hegemónicos, modelos excluyentes de la diversidad y la diferencia como elemento constitutivo de la misma realidad escolar y social.

\section{PRINCIPALES FACTORES QUE INCIDEN EN LA MOVILIDAD SOCIAL EDUCATIVA, DIFICULTADES DE ACCESO, DISPARIDAD EN LA CALIDAD Y PERTINENCIA}

Para la OCDE la movilidad educativa está relacionada con la distribución de ingresos y la movilidad dentro del sistema educativo, tanto de los padres como de los hijos, según la misma entidad, estos factores generan "movilidad de la movilidad social" teniendo en cuenta siempre variables como persistencia y regresión dentro del sistema. El mismo ente declara que en América Latina se ha venido dando un fuerte cambio en la expansión escolar, que en el caso Colombiano ha impactado con más fuerza a los grupos poblacionales de bajos recursos y mucho menos en los de ingreso medio, lo que genera inequidad diferencial por sectores económicos. No obstante la movilidad educativa y social en Colombia y la expansión educativa en América latina sigue estando muy por debajo de la de los países OCDE en el mundo. El análisis concluye que los altos desempeños académicos están relacionados con alta movilidad social, pero para el caso colombiano se destaca como excepcional el hecho que la movilidad social no solo depende del logro sino del entorno social 
lo que implica directamente no solo estatus socioeducativo y cultural de las familias sino el estatus socioeconómico de las mismas, representados por cantidad, calidad y distribución de ingresos ${ }^{3}$.

La mayoría de los estudios e informes sobre movilidad social y educativa en Colombia reportan que es la madre la que tiene mayor efecto en la determinación de logro educativo, incluida la posibilidad de educación superior (Galarza y Beltrán, 2004). En países como el nuestro donde la inequidad de género evidencia para ellas menores salarios, menores oportunidades de accesos laboral y de movilidad en los diferentes niveles del sistema educativo, así como, bajos logros en competencias científicas y matemáticas ${ }^{5}$, no así en competencia lectora ${ }^{6}$, implica poner en discusión las necesidades educativas de los géneros en primer renglón como elemento fundamental para disminuir la inequidad educativa. Si sumamos a esto, la baja asistencia y permanencia en el sistema educativo de las mujeres en instituciones públicas y rurales, tenemos otra gran preocupación en tremimos de equidad y sus posible repercusiones en movilidad social por genero lejos de los centros urbanos y en sectores poblacionales de bajos recursos, esto es clave si aceptamos la estrecha relación de la movilidad social y educativa de la madre con respecto a movilidad de los hijos.

El que padre y madre tengan primaria completa aumenta la posibilidad de que los hijos tengan formación universitaria completa y/o postgrados, si además los padres tienen educación secundaria el efecto es aun mayor, eso indican las estadísticas, pero ¿qué se sabe de la calidad de dicha formación en los padres y la repercusión en los hijos? Sobre todo si tenemos en cuenta que no necesariamente el tener credenciales académicas garantiza la movilidad social aquí influye también el nivel de logro y el éxito académico obtenido dentro del sistema educativo, entre otros.

3. El informe PISA presentado por el ICFES en el 2010, compara las cuatro ediciones del estudio en el mundo y concluye que en Colombia los factores socioeconómicos dan cuenta del 16,6\% de la varianza en los puntajes promedio de los estudiantes en lectura. El 83,4\% restante se debe a otras causas. Lo que complejiza el análisis para el caso colombiano y el número de variables a tener en cuenta.

4. En el estudio presentado por los investigadores de la universidad javeriana se encuentra, por ejemplo, que si el padre tiene educación primaria completa aumenta la probabilidad de que el hijo o la hija tengan educación postsecundaria, mientras que si es la madre, dicho incremento es casi tres veces mayor. Una importante excepción es en la probabilidad de que los hijos tengan educación postuniversitaria, sin embargo, la mayor influencia de la madre se nota claramente en el caso de la educación universitaria completa y otras. (Galarza y Beltran 2004).

5. En 35 países que aplicaron en la prueba PISA 2009 los resultados de los niños son mayores que los de las niñas, en cinco países sucede lo contrario y en los demás las diferencias no son significativas. Colombia es el país con la brecha más amplia en el mundo (32 puntos) a favor de los hombres. El promedio de los varones es de 398 y el de las mujeres de 366. Entre las naciones latinoamericanas, Chile y Colombia tienen las brechas más amplias a favor de los niños. (fuente ICFES 2010, informe PISA 2009).

6. El último informe de la aplicación SERCE para América latina confirma diferencias a favor de las niñas en el área de Lectura y a favor de los niños en Matemática y ciencias en la gran mayoría de los países. (fuente Informe SERCE - Segundo estudio Regional Comparativo y Explicativo 2008). 


\section{INEQUIDAD ENTRE NIVELES DEL SISTEMA EDUUATIIYO}

Recientemente Bernard Hugonnier?, señalaba que las políticas educativas, en términos generales, se debaten entre las que invierten en los estudiantes y grupos poblacionales más capaces, de tal forma que se alcance la formación de elites que compensen la inversión con desarrollo de talento local en diversos niveles y las políticas que buscan la movilidad educacional, igualando las oportunidades en educación e incluyendo masivamente en las instituciones educativas, buscando así, un bienestar generalizado. El problema de esta aparente polarización en la política para América latina, se centra en la atención sobre el segundo propósito, privilegiando el aumento de la cobertura en todos los niveles educativos frente a la calidad educativa de los mismos; priorizando la construcción y mejoramiento de la infraestructura frente a la adecuación y cualificación de los procesos educativos fundamentales del sector; consolidando Sistemas Nacionales de calidad de estudiantes e instituciones que dejan por fuera la evaluación de los docentes, de los programas curriculares y del impacto en el desarrollo de competencias y habilidades básicas de los mismos. Estas políticas para el caso colombiano en el último periodo de gobierno, presupone ingenuamente que el aumento en la conectividad del sector educativo genera pertinencia para la innovación y la productividad ${ }^{8}$, asumen ligeramente que aumentar los contenidos educativos virtuales, así como, el uso de las nuevas tecnologías en los currículos académicos, garantiza los procesos de mediación fundamentales en competencias y habilidades básicas. Otro aspecto que ha privilegiado la política educativa es la modernización de las entidades nacionales departamentales y locales mediante sistemas unificados de información, estas por sí mismas, no garantizan igualdad y oportunidad para el sector educativo. Países como Finlandia le apostaron a desarrollar al mismo tiempo políticas igualitarias y políticas para los de mayor avance, logrando movilidad educativa sin precedentes. Si los procesos de oportunidad discriminan a los estudiantes e instituciones más desarrollados, si no se jaIonan los procesos de desarrollo en competencias

7. Director adjunto de Educación de la Organización para la Cooperación y el Desarrollo Económico (OCDE) quien participó en el panel auspiciado por el MEN "El rol de las familias y la educación como factor de movilidad social". En el año 2010

8. Esta idea seguramente proviene del análisis obtenido por el ICFES del informe PISA 2009 para Colombia, en donde se señala que se encuentra ciertas relaciones entre los recursos del establecimiento educativo y los puntajes de los estudiantes Colombianos en lectura. Allí se menciona que los alumnos que están por debajo del nivel 2 en lectura (es decir que hacen lectura literal) tienen menos computadores en sus colegios; mientras que los ubicados en niveles más altos correlaciona con una mayor proporción de computadores, numero de ordenadores y acceso a internet. Esta correlación trasladada sin mayor reflexión pedagógica a la política pública, provee de discurso político eficiente y visible a gobernantes y entidades para estimar aumento en la calidad educativa a través de dotación en computadores y acceso a internet, sin mayores discusiones sobre el papel de la mediación de los mismos. Expectativas similares se vivieron con los programas de educación por radio y televisión de los cuales ya conocemos resultados positivos y negativos en el país. 
comunicativas, cognitivas, éticas y sociales a niveles superiores, se condena a las poblaciones y a las regiones a una nivelación por lo bajo, a distribuir la falta de oportunidades en sectores más amplios de la población sin movilidad interna entre los que están por debajo del logro local, regional e internacional, jalonando hacia abajo los que podrían estar en niveles medios y superior.

Otro aspecto que contribuye a la inequidad educativa esta asociado a la baja unicidad de las políticas y estrategias de atención y cobertura de los diferentes niveles en el sistema educativo colombiano, existen serias diferencias entre la atención, cobertura y calidad de la primera infancia con respectos a básica, media técnica y educación superior en el país ${ }^{9}$. "Una política de equidad debe tener un fuerte carácter preventivo más que correctivo de problemas que ya han hecho aparición, por ello es fundamental invertir más en políticas de educación y cuidado de la primera infancia y educación de los padres, ya que los niños y niñas inician la educación formal en condiciones muy desiguales" (Blanco Guijarro, 2008). Según el informe Atención integral a la primera infancia en Colombia "estrategia de País 2011-2014 del BID", el 9\% los niños y niñas menores de 5 años, se encuentran en situaciones de pobreza e indigencia en mayor nivel que el resto de la población, es decir que si el 46\% de los colombianos se encontraban en situación de pobreza y 17,8\% en situación de indigencia, los niños menores de 5 años alcanzaban el 48\% la pobreza y el $21 \%$ de indigencia para el periodo analizado. El mismo informe destaca que para niños y niñas menores de 5 años la cobertura educativa a nivel nacional está alrededor del 50\% para el año 2010, de este 50\%, el 97\% recibe atención del ICBF mediante hogares comunitarios. Los informes nacionales e internacionales coinciden que esta baja asistencia obedece, a la baja oferta educativa cercana a los lugares de residencia de los menores y a la poca necesidad que ven los responsables de los niños de llevarlos a un centro educativo y/o de atención al menor, al menos en los primeros años de vida (BID 2010). Los niños y niñas beneficiaros de los diversos programas de atención a la primera infancia, varían según las condiciones político administrativa de cada región en Colombia. La desigualdad se intensifica por diferencias de género, región, sector público o privado y diferencias etarias como "extraedad" entre otros.

Para la educación básica y media la situación es diferente, la fuerte mediación internacional ${ }^{10} \mathrm{y}$ la reciente preocupación nacional por el tema en este sector ha obligado a ampliar la cobertura del sistema educativo nacional y departamental como política desde el Ministerio de Educación Nacional.

9. Solo el hecho de que leyes diferentes y entidades diferentes acompañen a las políticas del Ministerio de Educación Nacional en estos sectores, implica tensiones y ambivalencias en las políticas de calidad en las oportunidades, en la cobertura, en la distribución y atención de las poblaciones y las regiones.

10. En el documento "Metas y estrategias de Colombia para el logro de los Objetivo de Desarrollo del Milenio 2015 " hacia una Colombia equitativa e incluyente plantea líneas base, estrategias y metas cuantificables sobre los retos esenciales del desarrollo humano en Colombia. Participan entidades Nacionales e internacionales como el DNP, el MEN, la ONU, el CEPAL y el PNUD. 
La idea de universalizar la educación y logar cobertura para derrotar el analfabetismo ${ }^{11}$ ha obligado a disminuir algunas de las diferencias internas en el país no las fundamentales, estas medidas presionan a los diferentes programas de gobierno, a evitar que grupos o regiones se rezaguen o que las personas más pobres no tengan acceso al sistema. Pese a lo anterior Bogotá y otras grandes ciudades concentran el mayor número de población atendida, mientras que en la periferia (regiones costeras y municipios no certificados) la cobertura decae significativamente ${ }^{12}$. Cabe aclarar que a pesar de estos logros lo avances en resultados nacionales e internacionales siguen siendo muy bajos, no es correlacional el éxito relativo en cobertura con la obtención de logro en competencias propias de las instituciones escolares.

Al comparar la educación superior entre regiones y el centro del país y al comparar los promedios nacionales, nuevamente apareen serias diferencias tanto en número de instituciones educativas a nivel superior como en población atendida. La educación superior que involucra la formación de maestros, por ejemplo, está centralizada, solo las grandes ciudades albergan el $90 \%$ de las instituciones educativas a nivel superior, en las regiones la oferta es muy baja, condicionado la formación de profesionales y generando rompimientos entre los vínculos y la movilidad social entre básica y media de las regiones con los procesos de formación en pregrado y postgrado, obligando a la migración de estudiantes a las ciudades o la formación bajo la oferta existente en la región que en la mayoría no esta acreditada por el CNA, no es de calidad y en relación costo beneficio genera mayor inequidad. Si a esto se le suma la participación del sector público y privado en la educación superior se observa que el sector publico hace más presencia en los departamentos mientras que el sector privado tiene mayor oferta en las ciudades principales como Bogotá, el apoyo a los procesos de de formación en los departamentos está ligado directamente al Instituto Nacional de Formación Técnica Profesional (INFOTEP) y el SENA ${ }^{13}$.

11. Las metas del milenio para Colombia consisten en disminuir la Tasa de analfabetismo de $1 \%$ para personas entre 15 y 24 años. (Línea de base 1992: 3.77\%). Lograr una Tasa de cobertura bruta del 100\% para educación básica (preescolar, básica primaria, básica secundaria) y de 93\% para educación media. (Línea de base 1992: 76.08\% y 59.11\%, respectivamente), alcanzar en promedio, 10.63 años de educación para la población entre 15 y 24 años (Línea de base $1992: 7$ años de educación) y disminuir la repetición a 2.3\% en educación básica y media (Línea de base 1992: 6.1\%)

12. La oferta de instituciones educativas en básica y media es más alta en Bogotá alrededor de un $10 \%$ frente a otras regiones como por ejemplo el 3\% de oferta de instituciones educativas en el magdalena y menos del 1\% para Casanare, estos datos contrastan con la cantidad de matriculados, preguntándose por la eficiencia y disponibilidad de cupos en las regiones centro periferia. Fuente: Matrícula certificada por las secretarías de educación (2002). MEN-Sistema Nacional de Información de Educación Básica (SINEB) [2003-2009]

13. EI SENA y el INFOTEP también tienen políticas y estrategias particulares e intereses diferenciales al resto del país, su prioridad son las competencias laborales y la preparación técnica. Estos intereses los han Ilevado incluso a interferir en los PEls de las instituciones educativas en donde, gracias a acuerdos de cooperación con las instituciones educativas la media técnica se transforma rápidamente en un currículo para toda la institución que nutra rápidamente el mercado laboral. 
Por su parte los incrementos en las últimas décadas de las matriculas en universidades privadas, obedecen a la elevada flexibilidad del sector en horarios, matriculas y diversidad curricular, aspecto que aun poco viable en el sector público, lo que nuevamente genera inequidad y baja movilidad social precisamente en sectores de la población neurálgicos ${ }^{14}$. Nuevamente la cobertura de este sector y sus posibles crecimientos o avances en los últimos años dista mucho de los resultados internacionales o nacionales ${ }^{15}$ de calidad, en este caso la acreditación, no va más allá de cumplir con currículos y programas homologables a nivel local regional e internacional ${ }^{16}$.

INEQUIDAD EN OBTENCION DE LOGRO

\section{En las diversas pruebas nacionales (SABER} $5^{\circ}, 9^{\circ}, 11^{\circ}$ y Pro) e internacionales (PISA, TIMSS y SERCE $)^{17}$ se evidencia marcadas diferencias de sus resultados ${ }^{18}$, por estratos socioeconómicos ${ }^{19}$, por género ${ }^{20}$, por zona rural o urbana y por el sector, es decir, carácter público o privado ${ }^{21}$ de las instituciones educativas.

Por su parte y basados en sus informes las pruebas de PISA; TIMSS y SERCE ${ }^{22}$ se anima el debate sobre la calidad de la enseñanza y el aprendizaje de las ciencias, el lenguaje y la

14. Por ejemplo en el Departamento del magdalena existen 13 instituciones de educación superior, concentradas en Santa Marta, que registran el 87.9 \% de la matrícula total. Los municipios que le siguen en participación son Ciénaga (6.2\%), El Banco (1.1\%) Fundación (1.0\%) y Chivolo (1.0\%). La universidad del magdalena una de la más destacada en la región Caribe con buenos resultados en pruebas nacionales ECAES. Para otras regiones como el departamento del Casanare la situación no es diferente las instituciones de educación superior como la Fundación Universitaria Internacional del Trópico Americano (UNITROPICO) con sede en Yopal la capital, genera un segundo nivel de centralización de los procesos educativos en esta región. Ante la baja oferta del sector educativo en este nivel los profesionales o egresados de básica y media, entre ellos los futuros docentes de la región, tienen que buscar opciones educativas en departamentos cercanos como en la Universidad Pedagógica y Tecnológica de Tunja y la Universidad de Boyacá que cubren la demanda educativa de más de un departamento a ese nivel.

15. Recientemente se ha intentado medir tanto a nivel nacional CNA como a nivel internacional (Sapiens Research) el grado de participación que tiene las IES (Instituciones de Educación Superior) en: investigación medida a través de grupos e investigadores por Colciencias; el número de publicaciones en revistas indexadas en publindex y el número de postgrados aprobados por el MEN. Estos datos sirven como elemento de criterio y referente para ser incluidos en Ranking de calidad educativa. Estas clasificaciones no necesariamente evalúan resultados académicos de estudiantes o de programas, sino que están más cerca de la lógica de certificación que ha venido imperando el mercado empresarial mundial. En el caso de los Ranking internacionales para universidades, además de artículos publicados, grupos de investigación y número de estudiantes, se tiene en cuenta número de premios Nobel obtenidos, otros reconocimientos y número de veces que son citados trabajos o publicaciones de los centros educativos y sus investigadores.

16. Buena parte de este interés está asociado a la necesidad de ofrecer mercados a las "multinacionales académicas" de Estados Unidos, Europa (España principalmente) y en algunos casos América latina (México, Brasil y chile entre otros). Se ofrece doble titulación, movilidad internacional y cursos de verano como gancho para reclutar estudiantes en el sistema educativo superior principalmente, e el negocio es tan rentable, que buena parte de las IES privadas en Colombia han sido vendidas o han establecido nexos comerciales con universidades extranjeras que ven en Colombia un buen mercado, básicamente por la creencia en que la movilidad social está asociada a la superación de logro, lo que no se dice es que el aumento en la oferta de ciertos programas satura el mercado, generando proletarización de la profesión y bajos ingresos para esos profesionales. 
matemática en el país. Los enfoques y propósitos evaluativos de estas pruebas son diferentes ${ }^{23}$. Guardando las proporciones en estas pruebas internacionales se observa un fenómeno similar al ya descrito ${ }^{24}$, promedios por debajo a los países participantes para el caso de PISA y TIMSS, y brechas marcadas con respecto a América latina para el caso de SERCE ${ }^{25}$.

En Colombia los resultados las pruebas SABER 2009, que evaluaron el desempeño por competencias de los estudiantes de los grados $5 .^{\circ}$ y $9 .^{\circ}$, muestran que un alto índice de estudiantes se ubica en el nivel de desempeño insuficiente en lenguaje, matemáticas y ciencias naturales, con porcentajes en el grado $5 .^{\circ}$ del $18 \%$, el $35 \%$ y el $18 \%$, y en el $9 .^{\circ}$ del $15 \%$, el $20 \%$ y el $14 \%$, respectivamente. Por su parte, SABER
11 (Examen de Estado) muestra que los mejores desempeños de los estudiantes se encuentran en el área de lenguaje con 46,47 puntos como promedio nacional, mientras áreas como filosofía y física no alcanzan los 44 puntos. Aquí se observa inequidad de logro no solo por genero sector y zona sino además por ciclo del sistema deductivo, son muy bajos los resultados entre los cortes $5^{\circ}, 7^{\circ}$ y $9^{\circ}$ para el país, estas brechas se incrementan a medida que se analiza colegios públicos y privado, regiones rurales y urbanas.

En términos generales y teniendo el rendimiento de las pruebas de grado $11^{\circ}$, que son las que se aplican al cierra de la formación básica y media en el país, con miras al ingreso a la educación superior el rendimiento en la mayoría de las competencias está por debajo del 50\%. Excepto por la

17. Pruebas internas y externas aplicadas en la última década en el país en relación con las ciencias, su aprendizaje y su enseñanza.

18. Por regiones se evidencias diferencias significativas en los resultados mostrados en grandes ciudades con centros de producción, industrialización y urbanización (Bogotá, Medellín, Cali y otras ciudades importantes) y menores avances en regiones rurales y apartadas de la geografía nacional (choco, guajira, Caquetá, putumayo, etc.)

19. El ICFES reporta que los estratos 1 y 2 están por debajo 41 puntos del promedio nacional mientras que los estratos superiores por encima de 50 puntos del promedio. Es interesante que los estratos 3 se encuentra en promedio equilibrados a nivel nacional. ( ICFES, 2011)

20. En el informe presentado por el ICFES (2011) sobre los puntajes promedio y desviaciones estándar por género en el núcleo común del Examen de Estado para educación media. Se observa que entre el 2005 y el 2010, para los dos calendarios A y B se presentan menores diferencias (medias inferiores a 1 punto) entre niños y niñas en lenguaje y ciencias sociales, mientras que las mayores diferencias (medias entre 2,2 y1,6 puntos en promedio) se presentan en matemáticas y en ciencias naturales respectivamente. Esto indica que dentro del sistema escolar es necesario plante las diferencias de oportunidad que tienen los niños y las niñas en competencias básicas sin que necesariamente se plantee, como en décadas anteriores, la separación de instituciones masculinas y femeninas en el país. Las oportunidades se deben plantear desde el desarrollo y las posibilidades del mismo entre géneros y entre habilidades a edades distintas.

21. A pesar del aumento en los últimos años, en el calendario $A$, los estudiantes urbanos aumentaron un $15 \%$ y los rurales crecieron el 63\% con un aumento en la zona urbana de colegios oficiales del 17\% y privado en un $10 \%$. Estos datos representan una participación promedio de 66\% para los estudiantes urbanos oficiales, 25\% para los urbanosprivados, $8 \%$ para los rurales-oficiales y 1\% para los rurales privados. El mayor crecimiento se dio entre las instituciones oficiales rurales (65\%): y las instituciones privadas rurales. Esto demuestra que a pesar del incremento del sector no se observan cambios significativos de logro en las regiones por ejemplo el puntaje promedio en el núcleo común 
educción privada en las grandes ciudades (no es igual el comportamiento en zonas rurales o regiones de la periferia) el promedio de los estudiantes nacionales indica que el 50\%, aproximadamente de los estudiantes que intentan ingresar a la educación superior cumple con los mínimos estándares en competencias designados en los documentos y lineamientos nacionales. Siendo preocupante que cerca de un $30 \%$ de los estudiantes se ubique en el nivel inferior lo que deja a muy bajos rangos poblacionales en todo el país ocupando niveles altos, superiores o muy superiores en desempeños. Aspectos que correlacionan con los bajos resultados obtenidos en pruebas internacionales como PISA y TIMSS. Es diferente en el caso de las pruebas SERCE en donde en términos generales y después de Cuba, Colombia se ubica en los mejores puntajes

\section{LINEAMIENTOS, POLÍTICA Y ESTRATEGIAS QUE PUEDEN ATENDER LA INEQUIDAD}

\author{
a. Hacer énfasis, visibilizar y \\ mediar de las diferencias de \\ género encontradas en el país.
}

Detectada la relación que existe entre la movilidad social y educativa asociada a los niveles educativos de las madres en el núcleo familiar y observando con buenos ojos los resultados nacionales e internacionales en competencias comunicativas del genero. Es preciso diseñar y proponer programas que atiendan las diferencias entre género en el sistema educativo y que analicen los factores que inciden en las brechas evidentes en competencias científicas y matemáticas. Las diferencias

de los estudiantes de establecimientos oficiales urbanos de calendario A fue de 46,8; el de estudiantes de establecimientos privados urbanos de 50,4 puntos; el de los estudiantes de colegios oficiales rurales, 44,6 y el de los alumnos de colegios privados rurales, 50,5 En el caso de calendario B, la media de los estudiantes de instituciones privadas urbanas y rurales fue 49,4 y 53,9, respectivamente. (ver informe Resultados ICFES 2005 2010)

22. Una diferencia importante entre estas tres pruebas es que TIMSS permite analizar los currículos de ciencia y matemáticas en sus alcances, contenidos y organización, mientras que PISA intenta dar cuenta de la posibilidad de extrapolar conocimientos a diferentes contextos y aplicar aprendizajes. En el caso de SERCE permite la caracterización de los sistemas educativos frente a coberturas, tasas, condiciones de las escuelas y al igual que TIMSS permite hacer seguimiento de los currículos pero a otro nivel, se pregunta por los dominios conceptuales y los procesos cognitivos que están inmersos en los currículos de los países participantes. En todos los casos las evaluaciones además permiten obtener información de factores asociados al aprendizaje en estudiantes, docentes e instituciones. De los estudiantes permite indagar por las condiciones del hogar, de su entrono familiar y sociocultural dinámica e interacción en el aula percepciones y grados de satisfacción frente a las instituciones educativas, los compañeros y los profesores así como actitudes, percepciones y valores y usos del tiempo escolar y extra escolar. De los docentes da cuenta de las rangos de edad, niveles de formación profesional, experiencia laboral en el campo de la enseñanza, estrategias y modos de la forma como se realizan las prácticas de enseñanza, percepciones sobre el grado de preparación y formación, profesional, satisfacción laboral, niveles y frecuencia de lectura, utilización de textos escolares y utilización de otros recursos entre otros

23. TIMSS indaga por la relación entre el currículo formulado, el desarrollado y el logrado en términos de aprendizajes al comparar lo que los estudiantes de cuarto y octavo grado (básica primaria y secundaria) saben en ciencias y matemáticas; PISA indaga por lo que los adolescentes de 15 años pueden hacer con lo que saben, determinando en qué medida los jóvenes son competentes en lenguaje, ciencias y matemáticas para la vida futura, independientemente de su grado escolar y SERCE compara el rendimiento en ciencias, matemáticas, lectura y escritura en primaria de los niños y niñas latinoamericanos. 


\begin{tabular}{|c|c|c|c|c|c|c|c|}
\hline \multicolumn{2}{|c|}{ Tabla 1: Años de educación por edad y grupos de ingresos en Colombia (2008) } \\
\hline INGRESOS & PROMEDIO & $\mathbf{1 4 - 2 0}$ & $\mathbf{2 1 - 3 0}$ & $\mathbf{3 1 - 4 0}$ & $\mathbf{4 1 - 5 0}$ & $\mathbf{5 1 - 6 0}$ & $\mathbf{6 1 - 6 5}$ \\
\hline Bajo & 4.42 & 7.50 & 6.54 & 4.91 & 4.21 & 3.08 & 2.81 \\
\hline Medio & 6.28 & 8.57 & 8.42 & 6.97 & 5.98 & 4.33 & 3.37 \\
\hline Alto & 1.80 & 10.00 & 11.96 & 11.73 & 10.50 & 9.35 & 7.51 \\
\hline
\end{tabular}

Fuente: OCDE 2010

de género en Colombia son importantes y comentadas por todas las pruebas internacionales como casos excepcionales, estas diferencias de género marcan derroteros en el accesos y permanencia al sistema educativo en todo los niveles y determina las decisiones profesionales y laborales tanto como su movilidad. En un país donde las madres por diversos motivos han quedado a cargo de los hijos, en donde los ingresos para ellas disminuyen y donde las posibilidades educativas son precarias, es necesario poner en la agenda política tales necesidades de manera prioritaria.

\section{b. Políticas que atiendan primera infancia y a sectores vulnerables.}

No solo es lograr la cobertura en los primeros años y en los estratos 1,2 y 3 se requiere como señala el diagnostico, unificarla discusión pedagógica y política entre los currículos iniciales y los de básica primaria en el país. Es necesario reunir a los maestros y directivos de las escuelas y jardines, centros de atención infantil y entidades a cargo a diseñar políticas conjuntas y pertinentes para la enseñanza en niveles iniciales, el debate debe atender la disminución de la fragmentación escolar entre el preescolar y la básica, debe generar puentes pedagógicos y administrativos entre la primera infancia y los posteriores desarrollos de los niños y niñas en las escuelas y atender de manera prioritaria sobre todo a nivel rural y urbano marginal a la infancia que esta por fuera del sistema educativo (pensando en el desarrollo de habilidades, competencias y talentos) incluso estando asistiendo a centros de atención.

Se habla mucho de atender a los estratos 1,2 y 3 en calidad cobertura y pertinencia educativa, lo que muestran los estudios es que se ha sobre atendido, por lo menos en cobertura a los estratos

24. Llama la atención que a pesar de que en pruebas como PISA no hay diferencias significativas por género o son muy bajas entre los países, Colombia evidencia una de las brechas más amplias, 21 puntos de diferencia de los niños frente a las niñas muy por encima de países como Estados Unidos y Liechtenstein. Estos datos contrastan con algunas naciones que a diferencia de Colombia y Estados Unidos evidencian puntajes que benefician a las niñas; Jordania, Albania, Dubái, Qatar y Argentina en América latina son ejemplo de ello. Otros países con diferencias significativas a favor de los niños son Chile y México en América latina. (ICFES, 2010) 
1 y 2, lo que si se evidencia es el abandono del la población con ingresos medios, es decir la de menor crecimiento en Colombia como señala el informe de la OCDE "El logro de la expansión escolar ha sido más rápido para los pobres que para la clase media, y para la clase media más que para los ricos". Los años de educación por edad e ingreso siguen siendo muy bajos para las poblaciones de bajos recursos, pero el menor crecimiento se ha venido presentando en las poblaciones de ingresos medios. Estas diferencia se incrementan en rangos de edad superior al de la adolescencia en donde disminuyen los años de permanencia de los sectores 1,2, y 3 con respecto a los 4,5 y 6 .

Políticas como la de la secretaria de educación de Bogotá de ampliar las becas, subsidios y disponibilidad frente al acceso a la educación superior de los más pobres puede ayudar, pero es preciso revisar también la calidad y permanencia en todos los renglones del sistema educativo en las regiones

\section{c. Políticas pertinentes y coherentes de Formación y evaluación de docentes.}

En el caso de la formación y la evaluación de maestros, se pueden encontrar en la ley general los elementos claves para la acreditación de las facultades de educación y escuelas normales encargadas de los procesos de formación de maestros, acreditación de programas y evaluación de la carrera docente ${ }^{26}$ en diferentes niveles. El Decreto 272 de febrero de 1998 y el Decreto 3012 de 1997 reglamentaron las disposiciones sobre formación docente que estableció la Ley 115. A su vez y relacionado con las políticas de formación de maestros en Colombia, se ubica la evaluación de la calidad del sistema educativo y la evaluación particularmente de los docentes en formación y en ejercicio dentro del mismo sistema. Las instituciones educativas y sus actores están constantemente siendo evaluados en el Sistema Educativo Nacional sin mucho impacto en la calidad y pertinencia del sistema; los estándares en competencias básicas, el mejoramiento planes de estudio y el mejoramiento institucional son algunos de los indicadores con los que cuenta el sistema para evaluar la calidad de las instituciones educativas; los planes de mejoramiento institucional y los planes de apoyo territorial son instrumentos de seguimiento al cambio dentro de las instituciones a nivel regional pero todos estos instrumentos han caído en políticas burocráticas nacionales y locales dejando de

25. Colombia en pruebas PISA 2006 por ejemplo obtuvo un promedio de 402 en ciencias estadísticamente inferior en ese mismo año al promedio de los países OCDE de 498 puntos. Con respecto América latina también se observan diferencias interesantes; el promedio de Colombia está por debajo de Chile, Uruguay y México; se equipara a los de Brasil y argentina; y supera a Perú y Panamá (PISA 2006).

26. En la Ley 715 de 2001 se establece el Estatuto de Profesionalización Docente mediante el Decreto 1278 de 2002 , este reglamenta las evaluaciones para el ingreso a la formación docente, al escalafón docente, el ingreso al sistema de educación pública, los periodos de prueba de los docentes en el sistema educativo y las competencias funcionales y comportamentales de la profesión docente. Todos los resultados obtenidos de estas diversas evaluaciones supuestamente nutren los planes de mejoramiento del sistema educativo en el país. Pero en la práctica los sindicatos, el clientelismo y la burocracia del propio sistema no permiten que estas evaluaciones retroalimenten y depuren el sistema educativo nacional. 


\section{En Colombia los resultados las pruebas SABER 2009, que evaluaron el desempeño por competencias de los estudiantes de los grados 5. y 9.', muestran que un alto índice de estudiantes se ubica en el nivel de desempeño insuficiente en lenguaje, matemáticas y ciencias naturales.}

lado la importancia que puede brindar la información obtenida para la calidad de la educación. Las evaluaciones en las aulas, evaluaciones externas al aula como saber, Saber 11 y los ECAES así como la evaluación institucional de docentes, facultades de formación y el mismo sistema de escalafón nacional intentan determinar de manera directa e indirecta el desempeño de los profesores dentro del sistema educativo. Aun así todo este universo evaluativo ${ }^{27}$ no logra transformar las prácticas pedagógicas en las instituciones escolares, no logran impactar en la calidad y pertinencia educativa de los estudiantes del sistema ni de los docentes en formación en las universidades. Se requiere incentivar la profesión docente, retribuir económica y socialmente a los mejores docentes y evaluar de manera significativa la permanencia y desempeño de docentes, programas de formación y currículos escolares derivado de la información suministrada por los sistemas de evaluación del país. ¿Qué impacto ha tenido en los PEls, en los currículos y en las áreas los resultados de casi 10 años de evaluación de las pruebas Saber en el país? ¿Qué cambios sugieren los resultados de la evaluación institucional de los docentes en el nuevo estatuto docente, en la calidad y continuidad de maestros de los colegios y universidades estatales? ¿Qué impactos ha tenido en los programas de formación docente los resultados de los ECAES de los últimos años?

27. En la ley general de educación y de acuerdo al artículo 80, el MEN y el ICFES han estado en los últimos años consolidando un Sistema integral y unificado de evaluación de la calidad de la educación. Este sistema se compone de tres fuentes importantes de información: la autoevaluación de las instituciones educativas; la evaluación externa de estudiantes y la evaluación de docentes y directivos docentes. La evaluación de docentes y directivos está contemplada en las leyes 115 de 1994 y 715 de 2001. En la ley 115 se establece el Estatuto docente mediante el Decreto 2277 de 1979. Artículos 80 y 84 Ley 115 de 1994 


\section{d. Es necesario rescatar los principios que dieron origen al PEl en las Instituciones Educativas}

Uno de los impactos de la constituyente del 91 en el sector educativo fue el democratizar la escuela de tal manera que la ley 115 de 1994, entre otros aspectos, estableció las bases para la descentralización ${ }^{28}$ de la educación formal en el país ${ }^{29}$ mediante la formulación de los PEl (Proyecto educativo Institucional). Introdujo en el debate escolar, el respeto por los derechos humanos, el derecho internacional humanitario, el reconocimiento de la diversidad y del pluralismo ideológico, religioso y político como base fundamental en la formación de ciudadanos ${ }^{30}$.

Los mecanismo de representatividad y participación en la escuela quedaron expuestos al formularse los órganos de gobierno escolares que descentralizaron el poder de los directivos y docentes en las instituciones educativas, expresando la idea central de la constituyente referente a la formación de los nuevos ciudadanos. La carta magna asumió la idea universal del derecho educativo tal como se expresan en el artículo 26 de la declaración de los derechos humanos. De esta suerte, los Proyecto Educativos Institucionales (PEI), los Planes de formación docente y las políticas educativas en Colombia, adquirieron como prioridad la descentralización y la participación de las comunidades educativas a todo nivel ${ }^{31}$. Pero el PEl, los PRAEs, los Planes de formación docente y buena parte de las políticas educativas del país se asumen en los sistemas escolares como imposiciones centralizadas y verticales que distan mucho de las necesidades puntuales de la escuela, por ello, es necesario elevar la percepción en las instituciones educativas frente la necesidad de trabajar como colectivos que produce conocimiento, avanzar en la necesidad de retomar el debate pedagógico, crear grupos de discusión y construcción

28. El esquema descentralizado implica la existencia de una organización sectorial diferenciada, en la que cada nivel de gobierno (nacional, departamental, distrital y municipal) tiene unas competencias y responsabilidades concurrentes y complementarias con la educación; es decir, las autoridades de cada entidad territorial deben ejercer sus funciones de manera coordinada con las de los otros niveles de gobierno. Fuente DANE

29. En Colombia el proceso de descentralización se ha orientado a la entrega de competencias, relacionadas con el manejo de recursos y de personal, a los diferentes niveles de gobierno. Se ha tratado de una descentralización administrativa, que contempla la transferencia de autoridad, responsabilidad y recursos Fuente DANE

30. "El Proyecto Educativo Institucional (PEI) es la estrategia fundamental, ordenada por la Ley 115 de 1994 , para propiciar la transformación de las instituciones como ejes de desarrollo y mejoramiento de la calidad educativa; El proyecto educativo como proyecto de desarrollo humano e institucional es un proceso permanente de construcción colectiva, que conlleva al crecimiento y desarrollo escolar y social de las comunidades educativas" Decreto 180 de 1997

31. "Para lograr la formación integral del educando, cada establecimiento educativo deberá elaborar y poner en práctica un Proyecto Educativo Institucional en el que se especifiquen, entre otros aspectos, los principios y fines del establecimiento, los recursos docentes y didácticos disponibles y necesarios, la estrategia pedagógica, el reglamento para docentes y estudiantes, y el sistema de gestión, todo ello encaminado a cumplir con las disposiciones de la presente ley, y sus reglamentos". Artículo 73 (Ley 115 1994) 
Uno de los impactos de la constituyente del 91 en el sector educativo fue el democratizar la escuela de tal manera que la ley 115 de 1994, entre otros aspectos, estableció las bases para la descentralización28 de la educación formal en el país29 mediante la formulación de los PEl (Proyecto educativo Institucional) de trabajo colectivo y empoderar en la capacidad de movilizar conocimientos a través de la escuela por líneas adicionales a las tradicionalmente reconocidas. Reanimar el debate pedagógico como otrora lo hiciera la "expedición pedagógica" representante de un periodo significativo para los docentes del país, es uno de los factores que impulsaría la revalorización del PEl en las instituciones educativas.

Plantear la necesidad de trabajar por equipos interdisciplinares internos y externos en talleres, foros y encuentros durante el año escolar en constante acompañamiento equitativo con pares académicos y con agentes de los sectores productivos, puede aportar en dar significados y oportunidades para que en el debate se reconozcan los docentes expertos y noveles recién incorporados a las dinámicas de la escuela en temas educativos y pedagógicos, donde se aproveche la experiencia de la praxis de los unos y las ideas frescas de la academia de los otros en la transformación de la enseñanza en los diferentes niveles del sistema escolar. Discutir en torno a la fragmentación escolar; a la posibilidad de privilegiar el desarrollo de los individuos en sus diversas dimensiones e integrar estas discusiones al modelo pedagógico institucional y a las reflexiones sobre el PEI, permite entre otros, vincular el PEI con el SIE (sistema integral de Evaluación) en un proyecto colectivo institucional y general que ponga en el centro la pregunta sobre ¿quiénes son los estudiantes a quienes se desea formar?, ¿Qué tipo de sociedad y de egresados quieren las instituciones a también a nivel regional y local y no solo nacional? y ¿Cuál es el propósito de la educación en el contexto actual de la escuela? 
Discutir y reflexionar sobre los Sistemas Integrados de evaluación (SIE) ${ }^{32}$, permite de alguna manera incidir en el diseño los sistemas de logro de las instituciones, proponiendo una mirada más integral, y formativa de los mismos, que finalmente permita la transformación de las prácticas de evaluación en el sistema escolar. Si se vinculan dichas prácticas con las estrategias pedagógicas y didácticas desarrolladas con éxito en la misma y otras instituciones, permitirá fortalecer el trabajo pedagógico de manera transversal en los currículos locales de tal forma que realmente se desarrollen individuos y contextos éticos, comprometidos y conocedores de su realidad social, individuos competentes al utilizar las diferentes formas de arte y lenguaje como medio de comunicación permanente en la construcción de su tejido social local, regional y nacional.

Es necesario superar el trabajo individual en los docentes, en los currículos y en las instituciones educativas de todo nivel. El trabajo educativo en solitario no logra trascender la escuela, aun nos cuesta a los maestros la discusión colectiva, la construcción en equipo y la divulgación y distribución del conocimiento construido en la escuela (relaciones escuela comunidad, escuela sector productivo y escuela academia). Este punto es de vital importancia, mucho más ahora en donde la distribución, socialización y construcción colectiva de conocimiento en la búsqueda de proyectos educativos discutidos y apropiados marcará la diferencia entra la pertinencia, durabilidad y apropiación del esfuerzo que se realice en la construcción y consolidación de un proyecto escolar local y regional.

Es necesario buscar la cohesión de equipos de docentes por niveles escolares y por áreas, de manera que adecúen el PEl y el currículo de las diversas necesidades educativas, garantizando reuniones periódicas conclusiones. Es necesario en definitiva acompañar a las instituciones educativas para mejorar las posibilidades de sostenibilidad de su PEl a corto, mediano y largo plazo, más que venderles formulas acabadas y estáticas de política educativa consignadas en documentos sin "vida" institucional. Es necesario centrar la actividad política, educativa y pedagógica en la reconstrucción del PEl en cada una de las instituciones, es necesario conectar el PIE de los diferentes niveles del sistema educativo. Conectar el preescolar con la básica; la básica con la media y la media técnica y este último con la formación universitaria en un proyecto colectivo que vincule la región con la nación, lo local con lo universal, lo básico con lo trascendente, lo concreto con la abstracto y lo simple con lo complejo en una unidad diferenciada.

\section{PRIMERAS GONCLUSIONES}

Es necesario descentralizar y cualificar la distribución de los recursos destinados al sector educativo, atender la histórica fragmentación del currículo, de las prácticas educativas y de la relación escuela comunidad escuela

32. Decreto 1290 
universidad. Es necesario poner en dialogo la política pública del sector educativo en donde tensiones sobre pertinencia calidad y cobertura se resuelvan inteligentemente.

Es necesario replantear la Baja o nula reflexión pedagógica actual en el sistema educativo, disminuir el privilegio administrativo frente a las necesidades académicas de las institucioOnes, directivos y docentes, se requiere pasar de una política con prioridad a la atención social en el sistema escolar por la atención educativa al mismo y aumentar la participación y conciencia local de la problemática educativa mas allá de las cifras de cobertura. Todos los informes de secretarios de educación trabajan en indicadores sociales, con repercusiones en la asignación de gasto y rubro, son pocos o nulos los indicadores de avance pedagógico y si los hay no hay consecuencias en ninguno de los renglones del sector educativo.

Es necesario asumir políticas locales para la primer infancia, con énfasis no solo en cobertura sino en calidad educativa y articulación con la política y las necesidades de básica, es necesario que dicha política promueva los docentes de este sector, los capacite, los empodere y dignifique profesionalmente articulándolos de manera seria y responsable con el resto del sistema educativo, es necesario vincular la universidad y los programas de formación con todos los niveles del sistema educativo en la calidad de pares académicos con responsabilidades y reconocimientos salariales similares partiendo también de balances y desarrollos del sector que impacten la calidad y pertinencia
Se hace imprescindible atender la relación centro- periferia; urbano- rural, regional- local y publico privado en el sistema educativo teniendo en cuenta que la corresponsabilidad, el coliderazgo y el compromiso con proyectos colectivos son y serna la clave para superar la inequidad y las brechas evidenciadas en el país.

Es necesario pasar de políticas que atiendan de manera exclusiva los problemas de pobreza y distribución a políticas que atiendan la singularidad del sistema educativo en lo referente a pertinencia y calidad educativa. No basta con incluir a la población en el sistema educativo, es necesario democratizar la escuela en lo pertinente a la misma, es decir democratizar el acceso al desarrollo intelectual, ético, comunicativo y de acción de los sujetos en los diferentes niveles y no solo suplir necesidades sociales insatisfechas como alimentación, subsidios, transporte, kid escolar, subsidio de asistencia entre otros. Finalmente el derecho a la educación debe ser la prioridad de las relaciones educativas en las aulas, dicho derecho no se basa solo en la asistencia dentro del sistema educativo hace referencia a la movilidad de calidad dentro del mismo, donde se cualifiquen las relaciones, la participación y la promoción de los sujetos más que la asistencia a través de servicios sociales. 


\section{REFERENCIAS}

7) ALBORNOZ, M (2001) Política Científica y Tecnológica Una visión desde América Latina Revista Iberoamericana De Ciencia Tecnología E Innovación OEI, Número 1, Septiembre - Diciembre 2001

1)BOGOYA ET ALL. 2005 Segundo Estudio Regional Comparativo Y Explicativo (SERCE UNESCO) Oficina Regional de Educación para América Latina y el Caribe OREALC / UNESCO Santiago Con la colaboración del ICFES. Bogotá (Colombia)

DDNP. Plan Nacional de Desarrollo 2002- 2006. Bogotá. Imprenta Nacional de Colombia. 2003.

DDNP. Plan Nacional de Desarrollo 2006 -2010.Colombia.

DDNP. Plan Nacional de Desarrollo 2010 - 2014.Colombia.

》) GALARZA J.T. Y BERNAL, G L ( 2004). Educacion y Movilidad Social en Colombia Facultad de Ciencias Economicas y Administrativas Departamento de Economía Pontificia Universidad Javeriana

》) GIL, MONSALUD; F et all .2010. PISA y la competencia científica: Un análisis de las pruebas de PISA en el Área de Ciencias. RELIEVE, v. 16, n. 2, p. 1-17. http:// www.uv.es/RELIEVE/v16n2/RELIEVEV16n2_6.htm

DICFES 2010. Colombia en PISA 2009 Síntesis de resultados. Bogota

DICFES 2010 Resultados de Colombia en TIMSS 2007 Bogotá

DICFES 2010 Resultados de Colombia en TIMSS 2007 Resumen ejecutivo. Bogotá

DICFES 2011 Examen de Estado de la educación media. Resultados del período 2005 - 2010. Bogotá

DICFES. 2010. Exámenes de Estado de Calidad de la Educación Superior SABER PRO (ECAES) Análisis de resultados del período $2004-2008$

1) LOZANO FLÓREZ. 2008. La Evaluación De Docentes En Colombia: Una Práctica Instrumental Y Burocrática. Revista Actualidades pedagógicas, enero junio numero 051 bogota
1) MEN 2001 Informe Nacional Sobre El Desarrollo De La Educación En Colombia 46a. Conferencia Internacional De Educación (CIE). Ginebra Suiza, SEPTIEMBRE

DMEN 2009. Organización Del Sistema Educativo Conceptos Generales De La Educación Preescolar, Básica Y Media Dirección de Descentralización Subdirección de Fortalecimiento a las Secretarías de Educación Ministerio de Educación Nacional. Bogotá, Colombia, Mayo de 2009 www.mineducacion.gov.co

DMEN. 2003. La revolución educativa. Plan sectorial Ministerio de Educación Nacional 2002-2003.

D MEN. Plan Decenal de Educación 19962005 "La educación un compromiso de todos”. Bogotá, 2006.

D) OCDE. 2002. Muestra de reactivos empleados en la evaluación PISA 2000 Aptitudes Para Lectura, Matemáticas Y Ciencias. OCDE Organización Para La Cooperación Y El Desarrollo Económicos. Santillana aula XXI México

DRAMÍREZ et all 2006 La educación primaria y secundaria en Colombia en el siglo XX jtelleco@banrep.gov. co. Banco de la república Bogotá Colombia

D RUBIO et all 2010. Atención integral a la primera infancia en Colombia: estrategia de país 2011-2014. Nota sectorial para su discusión con las nuevas autoridades y actores del sector. BID banco interamericano de desarrollo. Nota técnica 244 división de protección y salud

\) UNESCO 2010. Saltos mundiales de Educación $7^{\circ}$ edición 82010/11 en web

D) UNESCO,. 2008. Los aprendizajes de los estudiantes de América Latina y el Caribe. Primer reporte de los resultados del Segundo Estudio Regional Comparativo y Explicativo SERCE. Organización de las naciones unidas para la educación, la ciencia y la cultura Oficina Regional de Educación para América Latina y el Caribe. Chile 